\title{
APPLICATION OF HILBERT'S PROJECTIVE METRIC ON SYMMETRIC CONES
}

\author{
KHALID KOUFANY
}

\begin{abstract}
Let $\Omega$ be a symmetric cone. In this note, we introduce Hilbert's projective metric on $\Omega$ in terms of Jordan algebras and we apply it to prove that given a linear transformation $g$ such that $g(\Omega) \subset \Omega$ and a real number $p,|p|>1$, then there exists a unique element $x \in \Omega$ satisfying $g(x)=x^{p}$.
\end{abstract}

\section{INTRODUCTION}

If $A$ is a positive linear transformation on $\mathbb{R}^{n}$, then the Perron Theorem says that there exists $x_{0} \in \mathbb{R}_{+}^{n} \backslash\{0\}$ such that for all $x \in \mathbb{R}_{+}^{n}$, $A^{n} x$ converges in direction to $x_{0}$, i.e. $\frac{A^{n} x}{\left\|A^{n} x\right\|} \rightarrow \frac{x_{0}}{\left\|x_{0}\right\|}$. It has been shown by Birkhoff [1] that the Perron Theorem can be considered as a special case of the Banach contraction theorem. Birkhoff's approach is to consider a projective metric in a cone of positive elements in a Banach space. This metric was first introduced by Hilbert [6] when studying hyperbolic geometry. We begin with the definition of this metric in a general setting.

Let $V$ be is a real Banach space and $C$ be a closed convex pointed cone, where pointed means that $C \cap-C=\{0\}$. Denote the corresponding interior by $\Omega$. The relation $\preccurlyeq$ is defined on $V$ by saying that $x \preccurlyeq y$ if and only if $y-x \in C$. Hence $(V, \preccurlyeq)$ is a partially ordered linear space and it is Archimedean, that is, if $n y \preccurlyeq y$ for all $n \in \mathbb{N}^{*}$, then $y \preccurlyeq 0$.

For $x \in V$ and $y \in \Omega$ we let

$$
M(x, y):=\inf \{\lambda \mid x \preccurlyeq \lambda y\},
$$

and

$$
m(x, y):=\sup \{\mu \mid \mu y \preccurlyeq x\} .
$$

Hilbert's projective metric is defined on $\Omega$ by

$$
d(x, y)=\log \frac{M(x, y)}{m(x, y)} .
$$

1991 Mathematics Subject Classification. 17C15, 32M15.

Key words and phrases. Hilbert's projective metric, symmetric cones. 
In the case of $\mathbb{R}_{+}^{n}$, Hilbert's projective metric is $d(x, y)=\log \frac{\max \frac{x_{i}}{y_{i}}}{\min \frac{x_{i}}{y_{i}}}$ where $x=\left(x_{1}, \ldots, x_{n}\right)$ and $y=\left(y_{1}, \ldots, y_{n}\right)$ are two vectors of $\mathbb{R}_{+}^{n}$.

The Hilbert projective metric may be applied to variety of problems involving positive matrices and positive integral operators. For example one can use it to solve some Volterra equations. It is also particularly useful in proving the existence of the fixed point for positive operators defined in a Banach space. In this way, it has been shown by Bushell [4] that Hilbert projective metric may be applied to prove that, if $T$ a real nonsingular $r \times r$ matrix, then there exists a unique real positive definite symmetric $r \times r$ matrix $A$ such that

$$
T^{\prime} A T=A^{2} \text {. }
$$

Notice that if $T$ is neither symmetric nor orthogonal the existence and the uniqueness of $A$ is not an elementary problem, even if $r=3$.

In this note we will formulate the Hilbert projective metric on symmetric cones in a way most convenient for our purpose using Jordan algebra theory and extend Bushell's Theorem to this class of convex cones.

\section{Preparatory Definitions}

We summarize here a number of basic definitions and results concerning symmetric cones. For more detailed discussion, see Faraut and Korányi [5].

Let $\Omega$ be an open convex cone in a Euclidean vector space $V$ of dimension $n$. Let $G(\Omega)$ be the group of linear automorphisms of $\Omega$

$$
G(\Omega)=\{g \in G L(V) \mid g(\Omega)=\Omega\} .
$$

Then $\Omega$ is said to be homogeneous if $G(\Omega)$ acts on it transitively. If $\bar{\Omega}$ is pointed, then $\Omega$ is said to be symmetric if it is homogeneous and self-dual.

A Euclidean Jordan algebra is a Euclidean vector space $V$ equipped with a bilinear product such that

$$
\begin{aligned}
x y & =y x \\
x\left(x^{2} y\right) & =x^{2}(x y) \\
(x y \mid z) & =(y \mid x z) .
\end{aligned}
$$

It is shown that the interior $\Omega$ of the set of squares in $V$ is a symmetric cone, and every symmetric cone is given in this way.

We define the (left) multiplication $L$ by $L(x) y=x y$ and the so-called quadratic representation $P$ by $P(x)=2 L^{2}(x)-L\left(x^{2}\right)$. For any $x \in V$, the endomorphisms $L(x)$ and $P(x)$ are self-adjoint. 
Example 2.1. The space $\operatorname{Sym}(r, \mathbb{R})$ of real symmetric $r \times r$ matrices is a Jordan algebra for the product $x \circ y=\frac{1}{2}(x y+y x)$ and it is Euclidean for the scalar product $(x \mid y)=\operatorname{Tr}(x y)$. The associated symmetric cone is the set $\Omega_{\mathrm{Sym}}$ of positive definite symmetric $r \times r$ matrices and $G\left(\Omega_{\mathrm{Sym}}\right)$ is the linear group $\operatorname{GL}(r, \mathbb{R})$. In this case, $P(x) y=x y x$.

The Euclidean Jordan algebra has and identity element denoted by e. Let $K(\Omega):=\{g \in G(\Omega) \mid g(e)=e\}=$, then $K(\Omega)$ is a maximal compact subgroup of $G(\Omega)$. Let $r$ be the rank of $V$. A Jordan frame $\left\{c_{1}, \ldots, c_{r}\right\}$ of $V$ is a complete system of non-zero orthogonal primitive idempotents :

$$
\begin{aligned}
& c_{i}^{2}=c_{i}, c_{i} \text { indecomposable, } \\
& c_{i} c_{j}=0 \text { if } i \neq j, \\
& c_{1}+\ldots+c_{r}=e .
\end{aligned}
$$

We denote by $\mathcal{J}(V)$ the set of all primitive idempotents of $V$. Suppose $V$ is simple. In other words, there is no non-trivial ideal in $V$. Then each element $x$ in $V$ can be written as

$$
x=k\left(\sum_{j=1}^{r} \lambda_{j} c_{j}\right), k \in K(\Omega)_{\circ}, \lambda_{j} \in \mathbb{R},
$$

where $K(\Omega)$ 。 is the identity component of $K(\Omega)$. The determinant is defined by $\operatorname{det}(x)=\prod_{j=1}^{r} \lambda_{j}$ and the trace form by $\operatorname{tr}(x)=\sum_{j=1}^{r} \lambda_{j}$. The real numbers $\lambda_{1}, \ldots, \lambda_{r}$ are the eigenvalues of $x$ and the spectral norm of $x$ is then defined by

$$
|x|=\sup _{i}\left|\lambda_{i}\right|
$$

In the case of $\operatorname{Sym}(r, \mathbb{R}),(2.1)$ corresponds to the polar decomposition (diagonalization) of symmetric matrices. The functions det and $\operatorname{tr}$ are respectively the usual determinant Det and trace Tr of matrices.

\section{HilberT's METRIC ON SYMMETRIC CONES}

If we consider the cone $\Omega_{\mathrm{Sym}}$ of real symmetric positive definite $r \times r$ matrices, then one can easily express the Hilbert projective metric (1.1) in terms of eigenvalues of elements of $\Omega_{\mathrm{Sym}}$. Indeed, if $A$ and $B$ are in $\Omega_{\mathrm{Sym}}$, then

$$
M(A, B):=\inf \{\lambda \mid \lambda B-A \preccurlyeq 0\}=\max _{\|x\|=1} \frac{(A x \mid x)}{(B x \mid x)},
$$


and

$$
m(A, B):=\sup \{\lambda \mid \lambda B-A \preccurlyeq 0\}=\min _{\|x\|=1} \frac{(A x \mid x)}{(B x \mid x)},
$$

which are respectively the greatest and the least eigenvalue of $B^{-1} A$. Observe that eigenvalues of the matrix $B^{-1} A$ are the same of matrix $B^{-\frac{1}{2}} A B^{-\frac{1}{2}}=P\left(B^{-\frac{1}{2}}\right) A$ (see Example 2.1 for this notation).

More generally, for symmetric cones, Hilbert's projective metric can be also formulated in terms of extremal eigenvalues : let $x$ and $y$ be in $\Omega$ and let $\lambda_{M}(x, y)>0$ and $\lambda_{m}(x, y)>0$ denote the greatest and the least eigenvalue of the element $P\left(y^{-\frac{1}{2}}\right) x \in \Omega$. Then one can prove the following (see [7, Thoerem 4.2])

Proposition 3.1. We have

$$
\lambda_{M}(x, y)=\max _{c \in \mathcal{J}(V)} \frac{(x \mid c)}{(y \mid c)},
$$

and

$$
\lambda_{m}(x, y)=\min _{c \in \mathcal{J}(V)} \frac{(x \mid c)}{(y \mid c)} .
$$

From this characterization one can easily prove the following

Lemma 3.2. If $x, y \in \Omega$ and $\alpha, \beta \geq 0$, then

(1) $\lambda_{M}(\alpha x+\beta y, y)=\alpha \lambda_{M}(x, y)+\beta$,

(2) $\lambda_{m}(\alpha x+\beta y, y)=\alpha \lambda_{m}(x, y)+\beta$,

(3) $\lambda_{M}(x, y) \lambda_{m}(y, x)=1$.

Proposition 3.3. If $x, y \in \Omega$, then the Hilbert metric of $x$ and $y$ is given by

$$
d(x, y)=\log \frac{\lambda_{M}(x, y)}{\lambda_{m}(x, y)}=\log \lambda_{M}(x, y) \lambda_{M}(y, x) .
$$

Proof. According to Proposition 3.1, we have

$$
\begin{aligned}
M(x, y) & =\inf \{\lambda \mid x \preccurlyeq \lambda y\} \\
& =\inf \{\lambda \mid \lambda y-x \in \bar{\Omega}\} \\
& =\max \left\{\frac{(x \mid c)}{(y \mid c)} \mid c \in \mathcal{J}(V)\right\} \\
& =\lambda_{M}(x, y),
\end{aligned}
$$

and

$$
\begin{aligned}
m(x, y) & =\sup \{\mu \mid \mu y \preccurlyeq \lambda x\} \\
& =\sup \{\mu \mid x-\mu y \in \bar{\Omega}\} \\
& =\min \left\{\frac{(x \mid c)}{(y \mid c)} \mid c \in \mathcal{J}(V)\right\} \\
& =\lambda_{m}(x, y) .
\end{aligned}
$$


Applying Lemma 3.2, we obtain easily the second equality of the proposition.

Remark 3.4. It follows from Lemma 3.2 and Proposition 3.3 that $d$ is constant on rays,

$$
d(\lambda x, \mu y)=d(x, y) \text { for } \lambda, \mu>0 .
$$

In particular, if $\mu>0$, then the map $\xi_{\mu}: x \mapsto \mu x$ is an isomery of $d$.

\section{Contractions of Hilbert's projective metric}

Let $x=\sum_{j=1}^{r} \lambda_{j} c_{j}$ be an element of $\Omega$. If $p \in \mathbb{R}$, we write $x^{p}$ for $\sum_{j=0}^{r} \lambda_{j}^{p} c_{j}$ and we state the following contraction result :

Proposition 4.1. Let $p \in \mathbb{R}$ such that $|p| \leq 1$. Then the map

$$
\omega_{p}: \Omega \rightarrow \Omega, x \mapsto x^{p}
$$

is a contraction of $d$, that is, for any $x, y \in \Omega$,

$$
d\left(x^{p}, y^{p}\right) \leq|p| d(x, y) .
$$

Proof. Let $x, y \in \Omega$. Then $\lambda_{M}\left(x^{-1}, y^{-1}\right)=1 / \lambda_{m}(x, y)$ and $\lambda_{m}\left(x^{-1}, y^{-1}\right)=$ $1 / \lambda_{M}(x, y)$. Hence the map

$$
\imath: \Omega \rightarrow \Omega: x \mapsto x^{-1}
$$

is an isometry of $d$. It suffices then to prove (4.2) for $0 \leq p \leq$ 1. We know that $x \preccurlyeq \lambda_{M}(x, y) y$, then by the Loewner-Heinz inequality, see [8, Corollary 9], we have $x^{p} \preccurlyeq \lambda_{M}(x, y)^{p} y^{p}$. Therefore $\lambda_{M}\left(x^{p}, y^{p}\right) \leq \lambda_{M}(x, y)^{p}$. Using the same arguments we prove that $\lambda_{m}\left(x^{p}, y^{p}\right) \geq \lambda_{m}(x, y)^{p}$. Thus $d\left(x^{p}, y^{p}\right) \leq p d(x, y)$.

\section{Completeness}

Proposition 5.1. $(\Omega, d)$ is a pseudo-metric space. In other words, for any $x, y, z \in \Omega$, the following holds :

(a) $d(x, y) \geq 0$

(b) $d(x, y)=d(y, x)$

(c) $d(x, z) \leq d(x, y)+d(y, z)$

(d) $d(x, y)=0 \Leftrightarrow \exists \lambda>0: x=\lambda y$

Proof. Let $x, y$ and $z$ be in $\Omega$. The first propriety of the proposition is obvious, since $\lambda_{M}(x, y) \geq \lambda_{m}(x, y)$.

(b) From Lemma 3.2 we obtain

$$
\begin{aligned}
d(x, y) & =\log \frac{\lambda_{M}(x, y)}{\lambda_{m}(x, y)} \\
& =\log \lambda_{M}(x, y) \lambda_{M}(y, x) \\
& =d(y, x) .
\end{aligned}
$$


(c) Let $c$ be a primitive idempotent of $V$, then according to Proposition 3.1. $0<\frac{(x \mid c)}{(y \mid c)} \leq \lambda_{M}(x, y)$ and $0<\frac{(y \mid c)}{(z \mid c)} \leq \lambda_{M}(y, z)$. Hence $\frac{(x \mid c)}{(z \mid c)}=$ $\frac{(x \mid c)}{(y \mid c)} \frac{(y \mid c)}{(z \mid c)} \leq \lambda_{M}(x, y) \lambda_{M}(y, z)$ and

$$
\lambda_{M}(x, z) \leq \lambda_{M}(x, y) \lambda_{M}(y, z) .
$$

Similarly we prove that

$$
\lambda_{m}(x, z) \geq \lambda_{m}(x, y) \lambda_{m}(y, z) .
$$

It follows then from (5.1) and (5.2) that $d(x, z) \leq d(x, y)+d(y, z)$. (d) If $d(x, y)=0$, then $\lambda_{M}(, x, y)=\lambda_{m}(x, y):=\lambda$ and all the eigenvalues of $P\left(y^{-\frac{1}{2}}\right) x$ are equal to $\lambda$. Therefore, $P\left(y^{-\frac{1}{2}}\right) x=\lambda e$ and $x=\lambda P\left(y^{\frac{1}{2}}\right) e=\lambda y$. Conversely, if $x=\lambda y$ where $\lambda>0$, then $P\left(y^{-\frac{1}{2}}\right) x=\lambda e$ and $\lambda_{M}(x, y)=\lambda_{m}(x, y)=\lambda$.

Let $S(V)$ be the unite sphere in $V, S(V)=\{x \in V|| x \mid=1\}$ with respect to the spectral norm introduced in (2.2).

Lemma 5.2. Let $x$ and $y$ be in $\Omega \cap S(V)$. Then

(a) $|x-y| \leq e^{d(x, y)}-1$.

(b) If $|x-y|<\lambda_{m}(y)$ then $|x-y| \geq \lambda_{m}(y) \tanh \left\{\frac{1}{2} d(x, y)\right\}$, where $\lambda_{m}(y)$ is the least eigenvalue of $y$.

Proof. Let $x, y \in \Omega \cap S(V)$ and let $c$ be a primitive idempotent of $V$, then by Proposition (3.1) we have

$$
\lambda_{m}(x, y)(y \mid c) \leq(x \mid c) \leq \lambda_{M}(x, y)(y \mid c)
$$

But $|x|=1$ and $|y|=1$, then there exists $c_{1}, c_{2} \in \mathcal{J}(V)$ such that $\left(x \mid c_{1}\right)=1$ and $\left(y \mid c_{2}\right)=1$, thus

$$
\lambda_{m}(x, y) \leq 1 \leq \lambda_{M}(x, y) .
$$

Let $c_{0}$ be a primitive idempotent of $V$ such that $|x-y|=\left(x-y \mid c_{0}\right)$, then using (5.4) we have

$$
\begin{aligned}
|x-y| & =\left(x \mid c_{0}\right)-\left(y \mid c_{0}\right) \\
& =\left\{\frac{\left(x \mid c_{0}\right)}{\left(y \mid c_{0}\right)}-1\right\}\left(y \mid c_{0}\right) \\
& \leq \frac{\left(x \mid c_{0}\right)}{\left(y \mid c_{0}\right)}-1 \quad \text { since }|y|=1 \\
& \leq \lambda_{M}(x, y)-1 \\
& \leq \lambda_{M}(x, y)-\lambda_{m}(x, y) \\
& \leq\left\{\lambda_{M}(x, y)-\lambda_{m}(x, y)\right\} \frac{1}{\lambda_{m}(x, y)} \\
& =e^{d(x, y)}-1 .
\end{aligned}
$$


Moreover

$$
\begin{aligned}
\lambda_{M}(x, y) & =\max _{c \in \mathcal{J}(V)} \frac{(x \mid c)}{(y \mid c)} \\
& =\max _{c \in \mathcal{J}(V)}\left\{\frac{(x-y \mid c)}{(y \mid c)}+1\right\} \\
& \leq|x-y| / \lambda_{m}(y)+1 .
\end{aligned}
$$

Now, if $|x-y|<\lambda_{m}(y)$, then $1-\frac{|x-y|}{\lambda_{m}(y)}>0$. Therefor, from (5.5) and Lemma 3.2, we have $\left(1-\frac{|x-y|}{\lambda_{m}(y)}\right) y \preccurlyeq x$. Hence

$$
\lambda_{m}(x, y) \geq 1-\frac{|x-y|}{\lambda_{m}(y)} .
$$

Finally, it follows from (5.5) and (5.6) that $|x-y| \geq \lambda_{m}(y) \tanh \left\{\frac{1}{2} d(x, y)\right\}$.

Proposition 5.3. $(\Omega \cap S(V), d)$ is complete metric space.

Proof. It is clear that if $x, y \in(\Omega \cap S(V), d)$ such that $d(x, y)=0$, then $x=y$. Thus $(\Omega \cap S(V), d)$ is a metric space.

Let $\left(x_{k}\right)_{k}$ be a Cauchy sequence in $(\Omega \cap S(V), d)$. Then from Lemma 5.2 . $\left(x_{k}\right)_{k}$ is a Cauchy sequence in $(V,|\cdot|)$ and then converges to an element $x \in \bar{\Omega} \cap S(V)$. In order to prove that $x$ is an element of $\Omega \cap S(V)$, we observe, using (5.2) that $\lambda_{m}\left(x_{i}\right)=\lambda_{m}\left(x_{i}, e\right) \geq \lambda_{m}\left(x_{i}, x_{j}\right) \lambda_{m}\left(x_{j}, e\right)=$ $\lambda_{m}\left(x_{j}\right) \lambda_{M}\left(x_{i}, x_{j}\right) e^{-d\left(x_{i}, x_{j}\right)}$. Therefor, from (5.4), we obtain $\lambda_{m}\left(x_{i}\right) \geq$ $\lambda_{m}\left(x_{j}\right) e^{-d\left(x_{i}, x_{j}\right)}$. Since $\left(x_{k}\right)_{k}$ converges in the spectral norm to $x,\left(\lambda_{m}\left(x_{k}\right)\right)_{k}$ converges to $\lambda_{m}(x)$. It follows that for a fixed large $j, \lambda_{m}(x)=$ $\lim _{i \rightarrow \infty} \lambda_{m}\left(x_{i}\right) \geq \alpha \lambda_{m}\left(x_{j}\right)>0$, where $\alpha>0$. Hence $x \in \Omega \cap S(V)$. Finally, it is easy to prove using the second proposition of Lemma 5.2 that $\left(x_{k}\right)$ converges to $x$ in $(\Omega \cap S(V), d)$.

\section{Application}

If $V$ is the Jordan algebra $\operatorname{Sym}(r, \mathbb{R})$, then $\Omega$ is the symmetric cone of positive definite matrices, and $G(\Omega)$ coincides with the linear group $\operatorname{GL}(r, \mathbb{R})$. Recall that in this case $G(\Omega)$ acts on $\Omega$ by : $t \cdot a=t^{\prime} a t$.

In [4, Bushell prove that for any $t \in \operatorname{GL}(r, \mathbb{R})$ and any integer $k \geq 1$, there exists a unique real positive definite matrix $a$ such that $t^{\prime} a t=a^{2^{k}}$. For a general symmetric cone we have the following

Theorem 6.1. Let $g \in G(\Omega)$ and $p \in \mathbb{R}$ such that $|p|>1$. Then there exists a unique element $a$ in $\Omega$ such that $g(a)=a^{p}$. 
Proof. Since $\imath: x \mapsto x^{-1}$ is an isometry of $d$, see (4.3), we can assume that $p>1$. For $x \in \Omega \cap S(V)$ we put $F(x)=\frac{1}{\left|g(x)^{\frac{1}{p}}\right|} g(x)^{\frac{1}{p}}$. Then $F$ maps $\Omega \cap S(V)$ into $\Omega \cap S(V)$. Recall that Hilbert's projective metric is constant on rays, see (3.4), then we have for $x, y \in \Omega \cap S(V)$,

$$
d(F(x), F(y))=d\left(\left(\omega_{1 / p} \circ g\right)(x),\left(\omega_{1 . p} \circ g\right)(y),\right.
$$

where $\omega_{1 / p}$ is the power map $x \mapsto x^{\frac{1}{p}}$. We have already noticed in (4.1) that $\omega_{1 / p}$ is a contraction of the metric $d$, hence

$$
d(F(x), F(y)) \leq \frac{1}{p} d(g(x), g(y)) .
$$

Moreover, the eigenvalues of $P\left(y^{-1 / 2}\right) x$ are the unique solutions of the characteristic equation $\operatorname{det}(\lambda y-x)=0$. But, using [5. Proposition III.4.3],

$$
\operatorname{det}(\lambda g(y)-g(x))=\operatorname{det}(g(\lambda y-x))=\operatorname{Det}(g)^{n / r} \operatorname{det}(\lambda y-x) .
$$

Thus the eigenvalues $P(y)^{-1 / 2} x$ and $P(g(y))^{-1 / 2} g(x)$ are the same and $g$ is also an isometry of $d$. Finally all this implies that $F$ is a contraction of the Hilbert metric $d$,

$$
d(F(x), F(y)) \leq \frac{1}{p} d(x, y) .
$$

Therefore by the Banach contraction mapping theorem and Proposition 5.3 the map $F$ has a unique fixed point, let say $u$. Then $u^{p}=$ $\frac{1}{\left|g(u)^{1 / p}\right|^{p}} g(u)$ and the element $a:=\left|g(u)^{1 / p}\right|^{p} u \in \Omega$ is the unique solution of the equation $g(x)=x^{p}$.

Corollary 6.2. Let $h \in G(\Omega)$ and $p \in \mathbb{R}$ such that $|p|>1$. Then there exists a unique element $a$ of $\Omega$ such that $h\left(a^{p}\right)=a$.

Final remark. The Hilbert original definition of the projective metric involved the logarithm of the cross-ratio of for points in the interior of a convex cone in $\mathbb{R}^{n}$. It would be interesting to define the Hilbert projective metric on symmetric cones using the generalized cross-ratio introduced in [2], see also [7].

\section{REFERENCES}

1. G. Birkhoff, Extensions of Jentzsch's theorem. Trans. Amer. Math. Soc., 85 (1957), 219-227. MR19:296a

2. H. Braun, Doppelverhältnisse in Jordan-Algebren. Abh. Math. Sem. Univ. Hamburg 32 (1968), 25-51

3. P. J. Bushell, Hilbert's metric and positive contraction mappings in a Banach space. Arch. Rational Mech. Anal., 52 (1973), 330-338. MR 49:1247 
4. P. J. Bushell, On solutions of the Matrix Equation $T^{\prime} A T=A^{2}$. Linear Algebra and Appl., 8 (1974), 465-469. MR 51:551

5. J. Faraut and A. Korányi, Analysis on Symmetric Cones. Oxford Mathematical Monographs, Clarendon Press, Oxford, (1994). MR 98g:17031

6. D. Hilbert, Über die gerade Linie als kürzeste Verbindung zweier Punkte. Math. Ann., 46 (1895), 91-96.

7. K. Koufany, Contractions of angles in symmetric cones. Publ. RIMS, Kyoto Univ. $38(2002), \mathrm{n}^{\circ} 2$.

8. Y. Lim, Applications of geometric means on symmetric cones. Math. Ann., 319 (2001), 457-468. MR 1819877

Institut Elie Cartan, UMR 7502 (UHP-CNRS-INRIA), Université H. Poincaré, B.P. 239, F-54506 Vandeuvre-lès-Nancy Cedex, France

E-mail address: khalid.koufany@iecn.u-nancy.fr 